\title{
EDITORIAL
}

\section{COVID-19 infections in current orthopaedic practice}

After spreading on the whole globe and also in Romania starting with February 2019, the coronavirus disease pandemic has begun to loosen its strength in some of the most affected countries, allowing people to get back to their lives, however, being conscious that it is a serious disease, that they need to be cautious about anytime they meet with other people or touch objects that have been touched by others. In addition, all the countries affected have started taking the long road back to normalcy, while taking into account that they will have to face many challenges and that things will not ever be the way they were before the pandemic, or at least not very soon.

What is of utmost importance at present is to prevent the further spread of COVID-19 and to limit the possibility of the occurrence of a "second wave".

It is a fact that the SARS-Cov-2 pandemic affected all the fields, starting with the economy and ending with medicine. Many of the medical fields were affected by the coronavirus disease. Such a field is Orthopaedics. As in the other medical fields, many studies have been undergone on subjects related to COVID-19, such as: resuming elective orthopaedic surgery during the COVID-9 pandemic, prognosis of COVID-19 infection in the elderly with associated hip fractures, etc.

As time went by, we realized that it is important that us, as orthopedists and orthopaedics surgeons have to reduce pathogen transfer during the reintroduction of elective orthopaedic surgical procedures, with a focus on preventing the spread of SARS-CoV-2 infection among patients and even more among the elderly population, since they are the ones at risk.

Thus, taking into account that the highest prevalence of fractures among the elderly population are the fragility hip fractures, that occur due to the fact that patients have multiple associated comorbidities that lead to high mortality rates both in the preoperative and the postoperative period, we have come to the conclusion that current strategies of self-isolation at home, without support from the community, will probably put them at a higher risk of accidental falling in home.

Moreover, in the context of the COVID-19 pandemic, a large number of elderly patients with fragility hip fractures are already infected with the novel coronavirus from family encounters or they are admitted to the ER where nowadays COVID-19 infections are preponderant and the transmission rate is high. 
At this point, our common goal is to identify patients with fractures and chronic pathologies with COVID-19 associated infection, to monitor their evolution and to identify treatment strategies. At present, there is no standard protocol for hospital admission or for treating elderly patients with fractures and COVID-19 associated infections. In addition, a protocol needs to be developed regarding the optimal treatment for this category of patients. Moreover, an algorithm also needs to be created for the diagnosis and treatment of these patients, which will not increase infestation and mortality rates of these patients and will help us in future pandemic events.

In conclusion, these resources, once available, will lead to the better understanding of the implications future pandemics will have in fragile patients and develop future coping mechanisms for these events. 